
creativecommons.org/licenses/by/3.0/), which permits unrestricted re-use, distribution, and reproduction in any medium, provided the original work is properly cited.

\title{
The validation of a computer-based food record for older adults: the Novel Assessment of Nutrition and Ageing (NANA) method
}

\author{
Claire M. Timon ${ }^{1}$, Arlene J. Astell ${ }^{2,3}$, Faustina Hwang ${ }^{4}$, Tim D. Adlam ${ }^{5}$, Tom Smith ${ }^{4}$, Lin Maclean ${ }^{6}$, \\ Daynor Spurr ${ }^{7}$, Sarah E. Forster ${ }^{7}$ and Elizabeth A. Williams ${ }^{7 *}$ \\ ${ }^{1}$ UCD Institute of Food and Health, University College Dublin, Belfield, Dublin 4, Republic of Ireland \\ ${ }^{2}$ Faculty of Medicine, Dentistry and Health, School of Health and Related Research, University of Sheffield, \\ Sheffield S1 4DA, UK \\ ${ }^{3}$ Ontario Shores Centre for Mental Health Sciences, Whitby, ON, Canada L1N 559 \\ ${ }^{4}$ School of Systems Engineering, University of Reading, Whiteknights, Reading RG6 6AY, UK \\ ${ }^{5}$ Bath Institute of Medical Engineering, Wolfson Centre, Royal United Hospital, Bath BA1 3NG, UK \\ ${ }^{6}$ Institute of Health and Wellbeing, University of Glasgow, Gartnavel Royal Hospital, Glasgow G12 OXH, UK \\ ${ }^{7}$ Human Nutrition Unit, Department of Oncology, Faculty of Medicine, Dentistry and Health, University of Sheffield, \\ Sheffield S1O 2RX, UK
}

(Submitted 11 December 2013 - Final revision received 3 October 2014 - Accepted 16 October 2014 - First published online 29 January 2015)

\begin{abstract}
Dietary assessment in older adults can be challenging. The Novel Assessment of Nutrition and Ageing (NANA) method is a touch-screen computer-based food record that enables older adults to record their dietary intakes. The objective of the present study was to assess the relative validity of the NANA method for dietary assessment in older adults. For this purpose, three studies were conducted in which a total of ninety-four older adults (aged 65-89 years) used the NANA method of dietary assessment. On a separate occasion, participants completed a $4 \mathrm{~d}$ estimated food diary. Blood and $24 \mathrm{~h}$ urine samples were also collected from seventy-six of the volunteers for the analysis of biomarkers of nutrient intake. The results from all the three studies were combined, and nutrient intake data collected using the NANA method were compared against the $4 \mathrm{~d}$ estimated food diary and biomarkers of nutrient intake. Bland-Altman analysis showed a reasonable agreement between the dietary assessment methods for energy and macronutrient intake; however, there were small, but significant, differences for energy and protein intake, reflecting the tendency for the NANA method to record marginally lower energy intakes. Significant positive correlations were observed between urinary urea and dietary protein intake using both the NANA and the $4 \mathrm{~d}$ estimated food diary methods, and between plasma ascorbic acid and dietary vitamin C intake using the NANA method. The results demonstrate the feasibility of computer-based dietary assessment in older adults, and suggest that the NANA method is comparable to the $4 \mathrm{~d}$ estimated food diary, and could be used as an alternative to the food diary for the short-term assessment of an individual's dietary intake.
\end{abstract}

Key words: Dietary assessments: Older adults: Technology: Validation

Older adults bear the greatest burden of chronic disease in the developed world, and diet is known to play a significant role in the risk and progression of many chronic diseases associated with ageing ${ }^{(1,2)}$. Understanding of the complexities of the dietary determinants of health and disease requires accurate dietary intake assessment methods. There are a number of challenges associated with accurate dietary assessment in older adults ${ }^{(3-5)}$; physical limitations may reduce older adults' ability and motivation to complete food diaries, and cognitive decline may hinder retrospective methods of dietary assessment. While nutritional screening tools ${ }^{(6,7)}$ are commonly developed with the older adult population in mind, older adults are seldom considered in the development of dietary assessment methodologies ${ }^{(8)}$.

In recent years, there has been an increased drive towards the application of information and communication technologies (mobile phones, personal digital assistant (PDA) and computers) in the development of novel dietary assessment methods ${ }^{(9-11)}$, typically using computer-assisted $24 \mathrm{~h}$ dietary recall- ${ }^{(12-16)}$, diet history- ${ }^{(17-19)}$ or FFQbased $^{(20-23)}$ approaches. These information and communication technology-based approaches offer great potential to reduce the cost, improve the accuracy and reduce both participant and researcher burden of dietary assessment ${ }^{(9,24)}$.

Abbreviations: MAFF, Ministry of Agriculture, Food and Fisheries; MPR, multiple-pass recall; NANA, Novel Assessment of Nutrition and Ageing. 
However, these technology-based methods have tended to have been developed specifically for children ${ }^{(15,20-22,25)}$ or for the general adult population ${ }^{(13,14,16,23)}$, and the particular requirements of the older adult population have not been specifically considered in their development. This may, in part, reflect the belief that older adults are reticent to use computers.

A number of studies have reported that older adults encounter difficulties with using the methods designed for the general adult population. For example, significantly poorer agreement was found between a Web-based $24 \mathrm{~h}$ dietary recall and the reference method in participants $>60$ years compared with middle-aged adults (age 48-60 years) ${ }^{(26)}$. Older adults also reported more difficulty than younger adults in using a touch-screen audio computer-assisted self-interview for the assessment of diet, health and lifestyle in a population of American Indians, and cited a desire for more instructions ${ }^{(19)}$. This evidence highlights the need for computer-based dietary assessment methods that are more appropriate for use with older adults.

The main aim of the Novel Assessment of Nutrition and Ageing (NANA) study was to develop and validate a novel computer-based food record method specifically for use with older adults. We used touch-screen computer-based technology plus a fixed webcam to develop a method for capturing dietary intake in older adults (NANA), and describe here the relative validity of the NANA method against a $4 \mathrm{~d}$ estimated food diary and biochemical markers of nutrient intake.

\section{Methods}

\section{Novel Assessment of Nutrition and Ageing method}

NANA is a touch-screen computer-based dietary assessment method that enables older adults to record and photograph the food and drink they are consuming. The method is based on a self-reported food record. The main differences between the NANA method and a traditional food record is the use of a computer to record dietary intake, the capture of a food photograph in real time and the subsequent determination of portion size by the nutritionist rather than the participant. The system described in the present study used a 15.6-inch EeeTop touch-screen (Asus EeeTop, model ET1610PT) computer and a Microsoft ${ }^{\circledR}$ LifeCam Cinema $^{\text {TM }}$ webcam (Fig. 1). Users entered their food and drink items through a series of touch-screen button selections, which allowed them to navigate from twelve high-level food groups (Fig. 2), through a hierarchical food tree structure, to the selection of an individual, detailed food or drink item. The hierarchical food tree was composed of over $1200 \mathrm{food} /$ drink options. Each button showed a textual description of the food item or category, along with a photograph to help users recognise which button to choose. Immediately before food consumption, users would enter each of their meal items. The system would then prompt the users to photograph their items by placing them within view of the system's webcam and pressing a button on the screen. The system's webcam was in a fixed location above the screen. Users could take as many photographs as required to get all of their items recorded. The system then prompted the users to have their food/drink, and to return to the system after they had finished. At this point, they were asked whether they had any leftovers, and if so, they were asked to photograph the leftovers as well. If a user ate outside of the home or forgot to record the items at the time of consumption, they could still enter the items after the fact, but instead of photographing the items, they were prompted to verbally describe the portion sizes and these descriptions were audio-recorded by the system. Where the users could not find the food/drink item they were looking for, an 'item not found' option allowed them to audio-record a verbal description of the item.

The touch-screen computers (with the NANA software installed) and webcam were provided and installed by the researchers in the participants' homes. For the majority of the participants, the system was placed in the kitchen, although some participants preferred to locate the system in the dining area. Participants were given structured personal training in the use of the system by one of the researchers and were provided with a NANA reference manual and a helpline telephone number. Participants in study 1 were visited within $3 \mathrm{~d}$ of installation to check that they were happy with how to use the system, and that there were no technical glitches with either the software or the hardware. For the majority of the participants, this visit was unnecessary, and for subsequent studies, this home visit was only provided if requested by the participant. Participants were instructed to use the NANA method to record all food and drink items consumed over varying durations (as detailed below). At the end of the recording period, the system was uninstalled from the participant's home and the data were analysed. Food/drink descriptions, food photographs and voice recordings were used by the nutritionist to enter the dietary intake data into WinDiets nutritional analysis software (version 2010; Robert Gordon University). The assessment of food portion sizes was based on pre-consumption images and any photographs of leftover meal/drink items.

\section{Study 1}

For study 1, forty-four older adults (age 65-86 years) were recruited from Sheffield, England, UK. Participants used the NANA method to record their dietary intake for a period of 3 weeks. A blood sample and $24 \mathrm{~h}$ urine were collected during the second week of NANA data recording. Overall, three $24 \mathrm{~h}$ multiple-pass dietary recalls were collected over the duration of the study: the first 1 week before the installation of the system; the second at the point of the NANA installation; the third 1 week after the NANA data recording period. Participants completed a $4 \mathrm{~d}$ estimated food diary 4 weeks after the end of the NANA data recording period.

The $24 \mathrm{~h}$ multiple-pass recall (MPR) is a method commonly used with older adults ${ }^{(27)}$, and so this was included in study 1 as a potential additional reference method against which the validity of the NANA method could be considered. However, the $24 \mathrm{~h}$ MPR recorded significantly higher nutrient intakes 


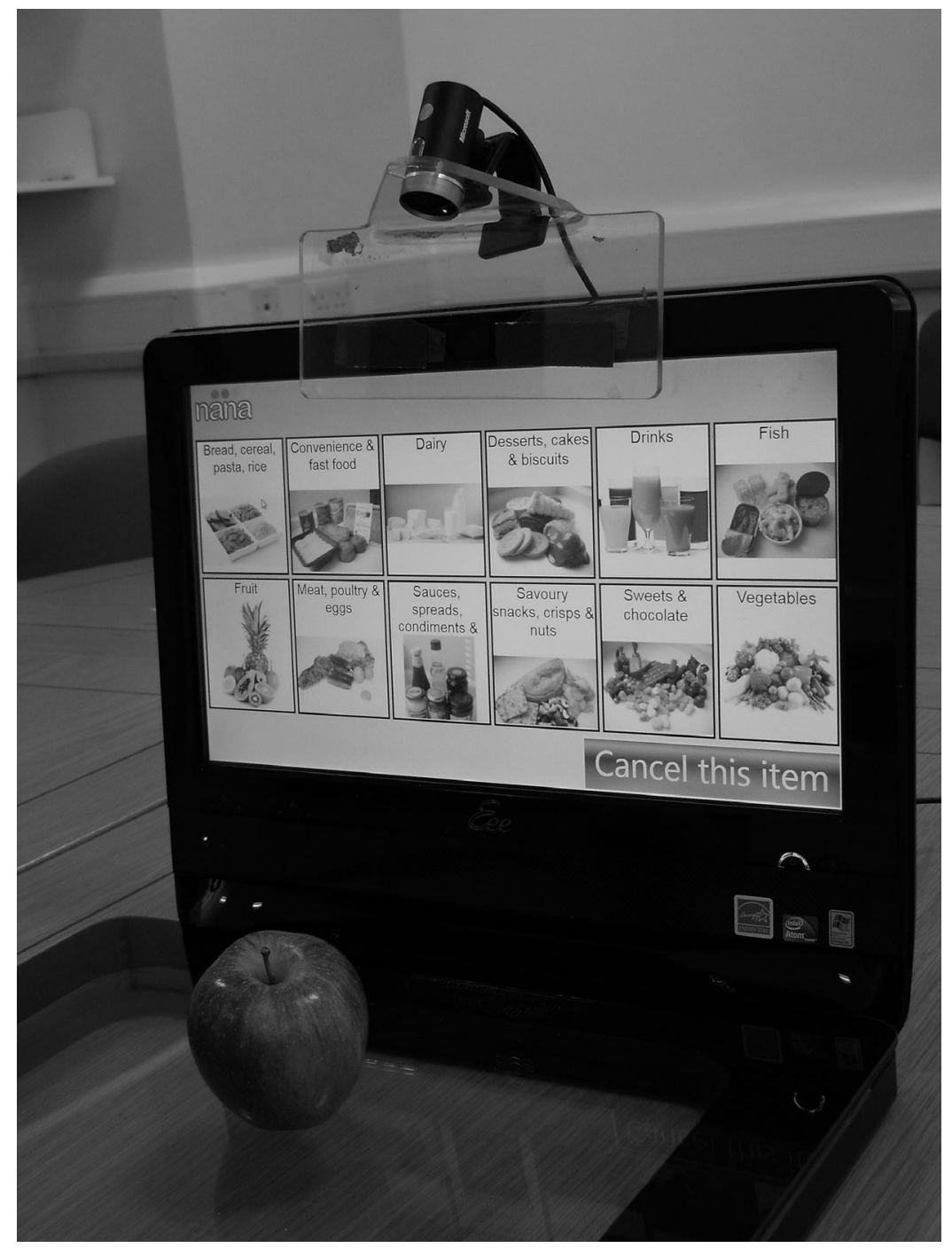

Fig. 1. Image of the Novel Assessment of Nutrition and Ageing (NANA) system.

than the food diary, and only weak-to-moderate associations were observed between nutrient intakes recorded by the $24 \mathrm{~h}$ MPR method and with both the $4 \mathrm{~d}$ estimated food diary and the NANA method (data not shown), and, in addition, no correlations were found between dietary intake and the independent biomarkers of protein and vitamin $\mathrm{C}$ intake. From this finding, the $24 \mathrm{~h}$ MPR was deemed to be a poorer reference method under these circumstances than the $4 \mathrm{~d}$ estimated food diary, and it was not used in subsequent studies. The reason for the poorer performance of the $24 \mathrm{~h}$ MPR compared with the food diary is unknown, but warrants further investigation in future studies.

\section{Study 2}

For study 2, nineteen older adults (age 68-86 years) were recruited from the residents of a retirement community and a neighbouring village in York, England, UK. The community is composed of individual homes and central buildings, providing a range of facilities including coffee shop, restaurant, hairdressers and care home. All the participants were living independently in their own homes. Participants recorded their dietary intake using the NANA method in their homes for $10 \mathrm{~d}$ and were asked to complete a $4 \mathrm{~d}$ estimated food diary 4 weeks after the NANA data recording period.

\section{Study 3}

For study 3, forty older adults (age 65-89 years), all living independently, were recruited from Sheffield, England, UK and St Andrews, Scotland, UK. Participants used the NANA method for $7 \mathrm{~d}$ on three different occasions at intervals of 4 weeks. A blood sample and $24 \mathrm{~h}$ urine were collected after the second NANA data recording period, and participants were asked to complete a $4 \mathrm{~d}$ estimated food diary 3 weeks after the third and the final NANA data recording period. In addition to the assessment of diet, software and hardware were added to the system to assess grip strength, self-reported mood, exhaustion, physical activity and proxy measures of cognitive status (to be reported elsewhere). 


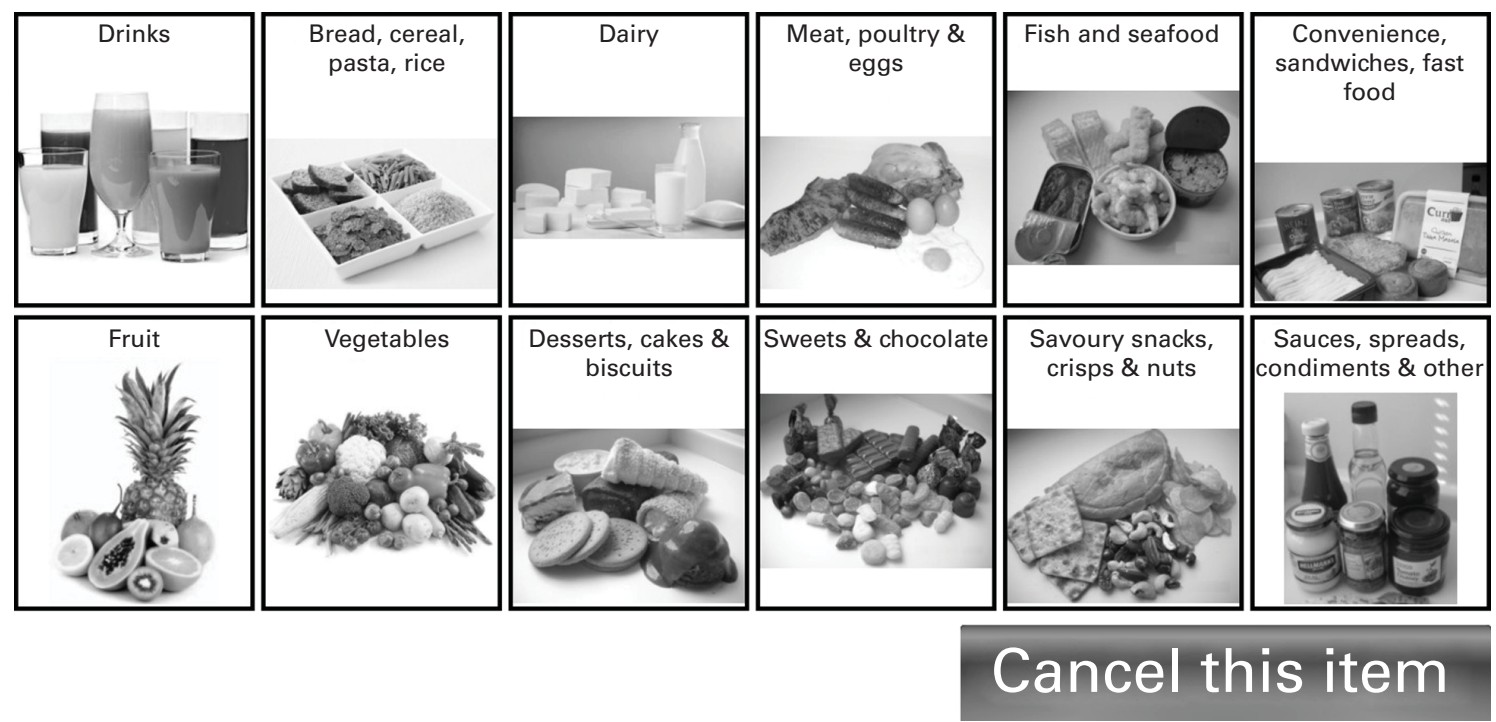

Fig. 2. Screenshot of the Novel Assessment of Nutrition and Ageing (NANA) software high-level food group options.

Minor modifications were made to the NANA dietary software between studies 1 and 3 such as the linking of foods commonly consumed together, and the addition of a 'favourites' facility to speed data entry. These changes did not significantly alter the dietary software interface or the user experience. In all the studies, participants were asked to consume their usual diet throughout the duration of the study. Participants were asked at the end of each study whether they had changed their eating behaviour and whether they had forgotten to record any items while using the NANA method.

\section{Ethical approval and consent}

The present study was conducted according to the guidelines laid down in the Declaration of Helsinki, and all procedures involving human subjects/patients were approved by the National Health Service (NHS) Fife and Forth Valley Research Ethics Committee (Ref.: 08/S0501/104) (studies 1 and 3) and the University of Sheffield Research Ethics Committee (Ref.: SMBRER 188) (study 2). Research governance was obtained from the Sheffield Health and Social Care, NHS Foundation Trust (Ref.: ZK71). Written informed consent was obtained from all the participants during a home visit at the start of the study. Participants in studies 1 and 3 were given a $\$ 100$ honorarium and those in study 2 a $£ 50$ honorarium to thank them for their participation in the research.

\section{Recruitment and inclusion and exclusion criteria}

Participants were recruited using volunteer databases, posters placed in community centres, advertisements placed in local newspapers, through older adult groups such as the University of the Third Age, and by word of mouth. Individuals who expressed an interest in the trial were sent an information sheet and were contacted by telephone by the researchers to discuss the study in more detail and to arrange an initial visit. Individuals were eligible for inclusion if they were aged 65 years or above, living in the community and able to provide informed consent. Individuals were excluded if they were residents of care home facilities, unable to provide informed consent, had a diagnosis of dementia or anticipated a deviation from their habitual diet during the study period.

\section{Dietary assessment}

4d estimated food diary. Participants were given a food diary and asked to record all the food, drinks and supplements consumed over a $4 \mathrm{~d}$ period (including one weekend day). Participants were also given a food portion size booklet containing photographs from the Ministry of Agriculture, Food and Fisheries (MAFF) food atlas to aid the assessment of food portion sizes ${ }^{(28)}$. Participants were asked to use the food photographs, household measures or weights as they found appropriate. Participants were interviewed at home by a researcher within 1 week of completion of the food diary, and the information was checked for clarity and completeness. Any anomalies or missing information was gathered by the researcher with the aid of the MAFF photographic atlas of food portion sizes ${ }^{(28)}$. Data from the $4 \mathrm{~d}$ estimated food diary were analysed for macronutrient and micronutrient content using WinDiets research software (version 2010; Robert Gordon University).

Novel Assessment of Nutrition and Ageing method. A custom software application, developed for use with the NANA software, was used by the nutritionist to view the photographs of the items consumed together with a textual description of the item collected via the food tree selection, and to listen to any audio recordings made. This software also included a digital catalogue of portion size photographs ${ }^{(28)}$ to facilitate the assessment of portion sizes. The nutritionist utilised all forms of data (photographs, textual description and audio recordings) in order to enter the items consumed into WinDiets research software. 
Data from $4 \mathrm{~d}$ of dietary intake, including one weekend day, collected using the NANA method were selected for the analysis. For studies 1 and 3 in which the NANA method was used over several weeks, the second data collection period was used for the analysis. Thus, for all the three studies, seven consecutive days of dietary intake data were considered for dietary analysis. The selection of $4 \mathrm{~d}$ for dietary analysis was largely determined by the need to include at least one weekend day. Consecutive days were selected for the analysis where possible; however, any day on which less than four eating/drinking occasions had been recorded was not used for the analysis. Days with gaps between eating/ drinking occasions of more than $5 \mathrm{~h}$ were also excluded from the analysis. This was to exclude any days of inadequate food recording or when food intake may have been significantly reduced due to ill health. The exception to these rules was when it was apparent that a lengthy period between NANA entries reflected time spent outside the home, in which case food selection and voice recordings were typically left at the end of the day. Ultimately, the judgement of the nutritionist was used to determine whether data entry for any particular day was complete and appropriate for selection, using number of meals, pattern of meal frequency and fluid intake to aid decision-making.

Biological samples were collected from the participants in studies 1 and 3 (as detailed below). Logistical limitations prevented the collection of samples from the participants in study 2.

Urine collection. Participants were instructed to collect their urine for $24 \mathrm{~h}$ immediately before attending the clinic for blood collection. Participants were provided with a plastic jug to help them collect their urine and a brown plastic 2-litre container to store their urine. Participants were instructed to discard the first urine sample of the day and to collect all subsequent urine produced for the next $24 \mathrm{~h}$ including the first urine sample produced the following day. The $24 \mathrm{~h}$ urine samples were collected in the clinic, mixed and the volume measured before two $20 \mathrm{ml}$ aliquots were taken and stored at $-20^{\circ} \mathrm{C}$ for subsequent analysis of urinary urea and creatinine concentrations.

Blood collection. Venous blood samples $(6 \mathrm{ml})$ were collected into lithium heparin tubes from each volunteer after an overnight fast. The samples were placed on ice and processed within $1 \mathrm{~h}$ of collection. Erythrocytes and plasma were separated by centrifugation. Plasma $(50 \mu \mathrm{l})$ was added to $450 \mu \mathrm{l}$ of $5 \%$ metaphosphoric acid to stabilise the plasma sample before the analysis of vitamin $\mathrm{C}$ concentration.

Biochemical analysis. Plasma ascorbic acid concentration was measured by a spectrophotometric method using the COBAS BIO Autoanalyser (Roche products) ${ }^{(29)}$. Urinary urea and creatinine concentrations were determined at the Department of Clinical Chemistry, Royal Hallamshire Hospital, Sheffield, using a centrifugal analyser (Roche/Hitachi COBAS c701 analyser). Urinary urea excretion was measured as an indicator of protein intake. Urinary urea excretion is at a constant proportion of total $\mathrm{N}$ excretion when an individual is consuming a normal Western diet, and is in energy balance ${ }^{(30)}$.

\section{Statistical analysis}

Statistical analysis was performed using SPSS (version 21, 2012; IBM Corporation). Levene's test was used to assess the homogeneity of the data across the three studies. Kolmogorov-Smirnov tests were used to examine the distribution of the data, and parametric or non-parametric tests were used accordingly for subsequent analysis. Pearson's and Spearman's correlation coefficients were used to examine the relationship between the nutrient intake data estimated using the NANA method and a $4 \mathrm{~d}$ estimated food diary. Paired Student's $t$ tests and Wilcoxon matched-pairs signedrank test were used to test for differences in the data collected using the NANA method and the $4 \mathrm{~d}$ estimated food diary. Bland-Altman analysis ${ }^{(31)}$ was used to assess the relationship between the NANA method and the $4 \mathrm{~d}$ estimated food diary.

\section{Results}

The characteristics (sex, BMI, age and technology use) of the participants in the three studies were broadly similar and the variance of the data (age, BMI, energy and macronutrient intake) was homogeneous, so the data from the three studies were pooled. A total of 103 older adults were initially recruited for the validation across the three studies; nine participants were excluded from the analysis, leaving a total of ninetyfour participants. Of those initially recruited participants, two withdrew from the study, both for personal reasons unrelated to the study, and a further seven participants (6.8\%) were excluded as they failed to fully utilise the NANA method as instructed.

The demographic characteristics of the participants in each of the three separate studies and for the pooled data are presented in Table 1. All the participants were British Caucasians. The mean age of the participants was 73 (range 65-89) years. Over $77 \%$ of the participants reported one or more medical conditions and reported the use of one or more prescribed medicines. Over a quarter of the participants reported having arthritis in their hands and/or having hearing problems. Nevertheless, few people experienced difficulties with everyday living such as reading a newspaper or preparing food, the exception being shopping for food that was reported to be a problem for $15 \%$ of the participants (the reason for this was not explored). A broad range of technology/computer skills were reported, with over $90 \%$ of all the participants having previously used a mobile phone or microwave, $86 \%$ having previously used a computer and nearly $80 \%$ having used the Internet.

Table 2 presents the mean daily energy and nutrient intakes using the NANA method and the $4 \mathrm{~h}$ estimated food diary, the correlation between the methods, the mean difference between the two methods, the limits of agreement (2SD), and the significance of the mean difference between the methods. There were highly significant correlations between the two reporting methods for all the macronutrients under study, ranging from a correlation of 0.879 for energy to 0.750 for protein. Significant, but weaker, correlations also existed for all the micronutrients investigated, ranging from 
Table 1. Demographic characteristics of the participants

(Number of participants and percentages)

\begin{tabular}{|c|c|c|c|c|c|c|c|c|}
\hline \multirow[b]{2}{*}{ Characteristics } & \multicolumn{2}{|c|}{ Study 1} & \multicolumn{2}{|c|}{ Study 2} & \multicolumn{2}{|c|}{ Study 3} & \multicolumn{2}{|c|}{ All } \\
\hline & $n$ & $\%$ & $n$ & $\%$ & $n$ & $\%$ & $n$ & $\%$ \\
\hline Caucasian & 40 & 100 & 18 & 100 & 36 & 100 & 94 & 100 \\
\hline \multicolumn{9}{|l|}{ Age (years) } \\
\hline Mean & \multirow{2}{*}{\multicolumn{2}{|c|}{$\begin{array}{c}72 \cdot 0 \\
65-85\end{array}$}} & \multirow{2}{*}{\multicolumn{2}{|c|}{$\begin{array}{c}75 \cdot 9 \\
68-86\end{array}$}} & \multirow{2}{*}{\multicolumn{2}{|c|}{$\begin{array}{c}71 \cdot 8 \\
65-89\end{array}$}} & \multirow{2}{*}{\multicolumn{2}{|c|}{$72 \cdot 7$}} \\
\hline Range & & & & & & -89 & & \\
\hline \multicolumn{9}{|l|}{ Sex } \\
\hline Female & 23 & 57.5 & 15 & $83 \cdot 3$ & 22 & $61 \cdot 1$ & 60 & $63 \cdot 8$ \\
\hline Male & 17 & 42.5 & 3 & $16 \cdot 7$ & 14 & 38.9 & 34 & $36 \cdot 2$ \\
\hline \multicolumn{9}{|l|}{ BMI $\left(\mathrm{kg} / \mathrm{m}^{2}\right)$} \\
\hline Mean & \multirow{2}{*}{\multicolumn{2}{|c|}{$\begin{array}{c}26 \cdot 4 \\
18 \cdot 3-35 \cdot 3\end{array}$}} & \multirow{2}{*}{\multicolumn{2}{|c|}{$\begin{array}{c}25 \cdot 8 \\
16 \cdot 7-39 \cdot 6\end{array}$}} & \multirow{2}{*}{\multicolumn{2}{|c|}{$\begin{array}{c}27 \cdot 9 \\
19.5-39.6\end{array}$}} & \multicolumn{2}{|c|}{$26 \cdot 9$} \\
\hline Range & & & & & & & & $-39 \cdot 6$ \\
\hline \multicolumn{9}{|l|}{ Medical history } \\
\hline Participants with $\geq 1$ medical condition & 32 & 80 & 13 & 72 & 28 & $77 \cdot 8$ & 73 & $77 \cdot 7$ \\
\hline Participants taking $\geq 1$ prescribed medicine & 30 & 75 & 14 & $73 \cdot 6$ & 29 & $80 \cdot 6$ & 73 & $77 \cdot 7$ \\
\hline \multicolumn{9}{|l|}{ Smoking status } \\
\hline Current smoker & 2 & $5 \cdot 0$ & 1 & $5 \cdot 6$ & 2 & $5 \cdot 6$ & 5 & $5 \cdot 3$ \\
\hline Ex-smoker & 15 & $37 \cdot 5$ & 6 & $33 \cdot 3$ & 7 & $19 \cdot 4$ & 28 & $29 \cdot 8$ \\
\hline Never smoker & 23 & 57.5 & 11 & $61 \cdot 1$ & 27 & 75 & 61 & 64.9 \\
\hline \multicolumn{9}{|l|}{ Factors affecting everyday living } \\
\hline \multicolumn{9}{|l|}{ Participants who reported } \\
\hline Having arthritis in their hands & 10 & 25 & 7 & 38.9 & 8 & $22 \cdot 2$ & 25 & $26 \cdot 6$ \\
\hline Hearing problems & 10 & 25 & 5 & $27 \cdot 8$ & 9 & $25 \cdot 0$ & 24 & $25 \cdot 5$ \\
\hline Difficulty in reading a newspaper & 0 & 0 & 0 & 0 & 1 & $2 \cdot 8$ & 1 & $1 \cdot 1$ \\
\hline Difficulty in preparing food & 0 & 0 & 1 & $5 \cdot 6$ & 0 & 0 & 1 & $1 \cdot 1$ \\
\hline Difficulty in shopping for food & 5 & $12 \cdot 5$ & 2 & $11 \cdot 1$ & 7 & $19 \cdot 4$ & 14 & 14.9 \\
\hline \multicolumn{9}{|l|}{ Technology usage } \\
\hline \multicolumn{9}{|l|}{ Participants who reported prior use of } \\
\hline A mobile phone & 40 & 100 & 16 & 88.9 & 34 & 94.4 & 90 & $95 \cdot 7$ \\
\hline A digital camera & 32 & 80 & 12 & $66 \cdot 7$ & 28 & 77.8 & 72 & $76 \cdot 6$ \\
\hline A microwave & 39 & $97 \cdot 5$ & 16 & 88.9 & 33 & 91.7 & 88 & 93.6 \\
\hline Self-service supermarket checkouts & 30 & 75 & 11 & $61 \cdot 1$ & 18 & 50 & 59 & $62 \cdot 8$ \\
\hline A computer & 35 & 87.5 & 14 & $77 \cdot 8$ & 32 & 88.9 & 81 & $86 \cdot 2$ \\
\hline \multicolumn{9}{|l|}{ Participants who reported } \\
\hline Home Internet access & 34 & 85 & 14 & $77 \cdot 8$ & 33 & 91.7 & 81 & $86 \cdot 2$ \\
\hline Being Internet users & 34 & 85 & 12 & $66 \cdot 7$ & 29 & $80 \cdot 6$ & 75 & $79 \cdot 8$ \\
\hline
\end{tabular}

0.572 for Fe to 0.265 for vitamin $B_{12}$. Differences between nutrient intakes were small but significant for energy, protein, alcohol, Fe, vitamins $\mathrm{A}, \mathrm{C}, \mathrm{B}_{6}$ and $\mathrm{B}_{12}$, and carotenoids. For all of these nutrients, the NANA method recorded lower intakes than the food diary, with intakes approximately $3 \%$ lower for energy and $5 \%$ lower for protein.

The energy and macronutrient intake data were further explored to assess the level of agreement between the two methods using Bland-Altman analysis. Fig. 3 shows the Bland-Altman plots of the difference between the two recording methods for total daily intakes of energy (Fig. 3(a)), protein (Fig. 3(b)), fat (Fig. 3(c)) and carbohydrate (Fig. 3(d)). For energy intake, the mean difference between the NANA and food diary methods was $-249 \mathrm{~kJ} / \mathrm{d}(59 \mathrm{kcal} / \mathrm{d})$ with lower and upper limits of agreement ( $2 \mathrm{SD})$ of -1887 to $1389 \mathrm{~kJ} / \mathrm{d}$. Although the mean difference between the reporting methods for energy intake was small, it was significantly different $(P=0.004)$. This illustrates the tendency for the NANA method to record marginally lower energy intakes than the food diary method. The mean difference for protein intake was $-3.68 \mathrm{~g} / \mathrm{d}$ with lower and upper limits of agreement of -26.04 to $18.68 \mathrm{~g} / \mathrm{d}$. Again, although the mean difference between the two methods was small, it was significantly different $(P=0 \cdot 002)$. The mean difference between the two methods for fat intake was small at approximately $-0.882 \mathrm{~g} / \mathrm{d}$ with limits of agreement of -24.2 to $22.4 \mathrm{~g} / \mathrm{d}$. The mean difference for carbohydrate intake was also small at $-5 \cdot 18 \mathrm{~g} / \mathrm{d}$ with limits of agreement of -68.98 to $58.62 \mathrm{~g} / \mathrm{d}$. There was no significant difference between the reporting methods for fat and carbohydrate intakes. The BlandAltman plots for energy and macronutrient intakes illustrate that there were very few outliers in the data, and most participants fell within the limits of agreement; there was also no apparent proportional bias, illustrating that the differences in intake between the two methods occurred at random across the range of intakes.

Urinary urea excretion was measured in the urine samples of the seventy-six participants in studies 1 and 3. Blood samples were collected from seventy-four of these participants; however, eighteen participants were excluded from the analysis of the relationship between plasma ascorbic acid and dietary vitamin $\mathrm{C}$ intake as they reported consuming either vitamin $C$ or multivitamin supplements. Fig. 4 presents the scatter plots of the relationship between reported dietary intake and the independent biomarkers of nutrient intake. There were significant positive correlations between urinary urea and dietary protein intake recorded by both the NANA method ( $r$ 0.466, $P<0.0001$; Fig. 4(a)) and the food diary 
Table 2. Daily energy and nutrient intakes recorded by older adults using the Novel Assessment of Nutrition and Ageing (NANA) method and the $4 \mathrm{~d}$ estimated food diary

(Mean values and standard deviations)

\begin{tabular}{|c|c|c|c|c|c|c|c|c|c|}
\hline \multirow[b]{2}{*}{ Nutrients } & \multicolumn{2}{|c|}{ NANA } & \multicolumn{2}{|c|}{ Food diary } & \multirow{2}{*}{$\begin{array}{l}\text { Correlation } \\
\text { coefficient }(r)\end{array}$} & \multicolumn{2}{|c|}{ Difference } & \multirow{2}{*}{$\begin{array}{l}\text { Limits of } \\
\text { agreement† }\end{array}$} & \multirow[b]{2}{*}{$P$} \\
\hline & Mean & SD & Mean & SD & & Mean & SD & & \\
\hline Energy $(\mathrm{kJ} / \mathrm{d}) \ddagger$ & 7461 & $158 \cdot 3$ & 7709 & $176 \cdot 7$ & $0.879^{\star \star \star}$ & -249 & 819 & $-1887 / 1389$ & 0.004 \\
\hline Protein $(g / d) \ddagger$ & $73 \cdot 3$ & $15 \cdot 18$ & $77 \cdot 0$ & $16 \cdot 31$ & $0 \cdot 750^{\star \star \star}$ & -3.68 & $11 \cdot 181$ & $-26 \cdot 04 / 18 \cdot 68$ & 0.002 \\
\hline Fat $(g / d) \ddagger$ & 73 & $19 \cdot 0$ & 74 & $20 \cdot 4$ & $0.827^{\star \star \star}$ & -0.882 & 11.66 & $-24 \cdot 2 / 22 \cdot 4$ & 0.465 \\
\hline Saturated fat $(\mathrm{g} / \mathrm{d}) \ddagger$ & 27 & 8.6 & 27 & $8 \cdot 8$ & $0.752^{* * *}$ & $0 \cdot 180$ & $6 \cdot 134$ & $-12 \cdot 08 / 12 \cdot 44$ & 0.776 \\
\hline Carbohydrate $(\mathrm{g} / \mathrm{d}) \ddagger$ & 205 & 53.4 & 211 & $55 \cdot 7$ & $0.830^{\star * *}$ & $-5 \cdot 18$ & 31.90 & $-68 \cdot 98 / 58 \cdot 62$ & 0.119 \\
\hline NSP $(g / d) \ddagger$ & $13 \cdot 9$ & 4.68 & 13.5 & $4 \cdot 27$ & $0.768^{\star \star \star}$ & 0.355 & 3.08 & $-5 \cdot 80 / 6 \cdot 51$ & 0.266 \\
\hline Alcohol $(g / d) \S$ & 7.97 & $12 \cdot 62$ & $10 \cdot 5$ & 14.29 & $0.738^{\star \star \star}$ & -2.57 & 8.421 & $-19 \cdot 41 / 14 \cdot 27$ & 0.010 \\
\hline $\mathrm{Ca}(\mathrm{mg} / \mathrm{d}) \ddagger$ & 868 & $264 \cdot 7$ & 907 & $268 \cdot 2$ & $0.560^{\star * *}$ & -39.0 & $249 \cdot 9$ & $-538 \cdot 8 / 460 \cdot 8$ & 0.133 \\
\hline $\mathrm{Fe}(\mathrm{mg} / \mathrm{d}) \neq$ & $11 \cdot 3$ & $3 \cdot 18$ & $12 \cdot 0$ & 3.37 & $0.572^{\star \star \star}$ & -0.764 & 3.037 & $-7.50 / 5 \cdot 98$ & 0.017 \\
\hline Vitamin A $(\mu \mathrm{g} / \mathrm{d}) \S$ & 1032 & $875 \cdot 6$ & 1137 & $939 \cdot 8$ & $0.455^{\star * *}$ & -105 & $1218 \cdot 9$ & $-2332 / 2437$ & 0.044 \\
\hline Riboflavin (mg/d) $\ddagger$ & 1.80 & 0.635 & 1.81 & 0.591 & $0.448^{\star \star \star}$ & -0.016 & 0.645 & $-1 \cdot 03 / 1 \cdot 27$ & 0.811 \\
\hline Vitamin $B_{6}(\mathrm{mg} / \mathrm{d}) \ddagger$ & 1.83 & 0.591 & 1.94 & 0.539 & $0.560^{\star \star \star}$ & -0.106 & 0.492 & $-1.089 / 0.877$ & 0.039 \\
\hline Vitamin $B_{12}(\mu \mathrm{g} / \mathrm{d}) \S$ & 4.94 & 3.42 & $5 \cdot 84$ & $4 \cdot 16$ & $0 \cdot 265^{\star *}$ & -0.906 & $5 \cdot 129$ & $-11 \cdot 1 / 9 \cdot 35$ & 0.011 \\
\hline Folate $(\mu \mathrm{g} / \mathrm{d}) \ddagger$ & 268 & $108 \cdot 6$ & 286 & $109 \cdot 4$ & $0 \cdot 367^{\star \star \star}$ & -18.4 & $122 \cdot 67$ & $-263 \cdot 7 / 226 \cdot 9$ & 0.149 \\
\hline Vitamin C $(\mathrm{mg} / \mathrm{d}) \ddagger$ & $90 \cdot 4$ & $42 \cdot 4$ & 104 & $64 \cdot 1$ & $0.614^{\star \star \star}$ & $-13 \cdot 9$ & $50 \cdot 71$ & $-115 \cdot 3 / 87 \cdot 5$ & 0.009 \\
\hline Retinol $(\mu \mathrm{g} / \mathrm{d}) \S$ & 548 & $760 \cdot 8$ & 476.5 & $734 \cdot 1$ & $0.389^{\star \star \star}$ & 71.03 & $1077 \cdot 8$ & $-2084 / 2226$ & 0.264 \\
\hline Carotenoids $(\mu \mathrm{g} / \mathrm{d}) \ddagger$ & 2909 & $2023 \cdot 5$ & 4004 & $2902 \cdot 4$ & $0.435^{\star \star \star}$ & -1094 & 2721.4 & $-6534 / 4348.9$ & 0.000 \\
\hline
\end{tabular}

${ }^{\star *} P=0.01,{ }^{\star \star \star} P<0.001$.

$\dagger$ Lower and upper limits of agreement (mean difference $\pm 2 \mathrm{SD}$ ).

$\ddagger$ Energy and nutrient intake data were examined by Pearson's correlation coefficient and differences were estimated by paired Student's $t$ test.

$\S$ Nutrient intake data were examined by Spearman's correlation coefficient and differences were estimated by Wilcoxon matched-pairs signed-rank test.

method ( $r$ 0.563, P<0.0001; Fig. 4(b)). There was also a significant positive correlation between plasma ascorbic acid and dietary vitamin $\mathrm{C}$ intake recorded by the NANA method ( $r$ 0.294, $P=0.028$; Fig. 4(c)), but not with the food diary method $(r-0.001, P=0.996$; Fig. $4(d))$.

\section{Discussion}

There was generally good agreement between the NANA method and the $4 \mathrm{~d}$ estimated food diary for energy and nutrient intakes as shown by Bland-Altman analysis, and it is proposed that the NANA method is an appropriate alternative to the $4 \mathrm{~d}$ estimated food diary. When considering the validity of a novel dietary reporting method, most researchers report means and correlations and seldom consider agreement using Bland-Altman analysis. The present study demonstrated a good-to-moderate correlation between the NANA method and the $4 \mathrm{~d}$ estimated food diary for total energy intake ( $r$ 0.88), macronutrient intake (mean $r 0.80)$ and micronutrient intake (mean $r$ 0.47). The correlation between the NANA method and the reference method compares favourably with the results from other validation studies of technology-assisted dietary assessment ${ }^{(16,18,23,32)}$. Despite a relatively good agreement between the two methods as illustrated by BlandAltman analysis and highly significant correlations between the methods, there were small but significantly lower mean daily dietary intakes of energy $(-249 \mathrm{~kJ} / \mathrm{d})$ and protein $(-3.7 \mathrm{~g} / \mathrm{d})$ reported by the NANA method than by the food diary, reflecting a degree of apparent systematic bias. There were also significant differences for intakes of alcohol and a number of micronutrients. There are several possible reasons why significant differences between the two methods were observed; the most important being that the data were collected over two different recording periods. The studies were designed in this way so that the test method of dietary assessment was not influenced by the reference method ${ }^{(33)}$. Differences in the actual food consumed on the two occasions will, therefore, lead to differences in nutrient intake; this typically has the greatest impact on micronutrient intake as is evident from the data. The NANA method was employed first, and there may have been an effect of initial monitoring that resulted in participants reducing their intake. Approximately one-third of the participants reported that they had eaten less as a consequence of recording their intakes using the NANA method, citing that they were more aware of their consumption. The same question was not asked of the food diary method, although under-reporting and altered food consumption is commonplace with food records. Alternative explanations for the lower intake recorded by the NANA method are that participants using the NANA method may have failed to enter all the food items or may not have provided sufficient detail of forgotten items and foods consumed outside the home during audio recordings. A number of participants reported that snacks and drinks were the foods that they most commonly omitted from NANA recording and could explain the apparent difference between the two recording methods. It is also possible that the lower intake reporting is a result of the researcher being more accurate in the estimation of food portion sizes from photographs collected from the NANA system than the participant's own estimation using a combination of portion-size reporting methods with the food diary. It should also be remembered that the reference method is also subject to errors and bias, and differences may have arisen as a consequence of errors in the $4 \mathrm{~d}$ estimated food diary ${ }^{(34)}$. 

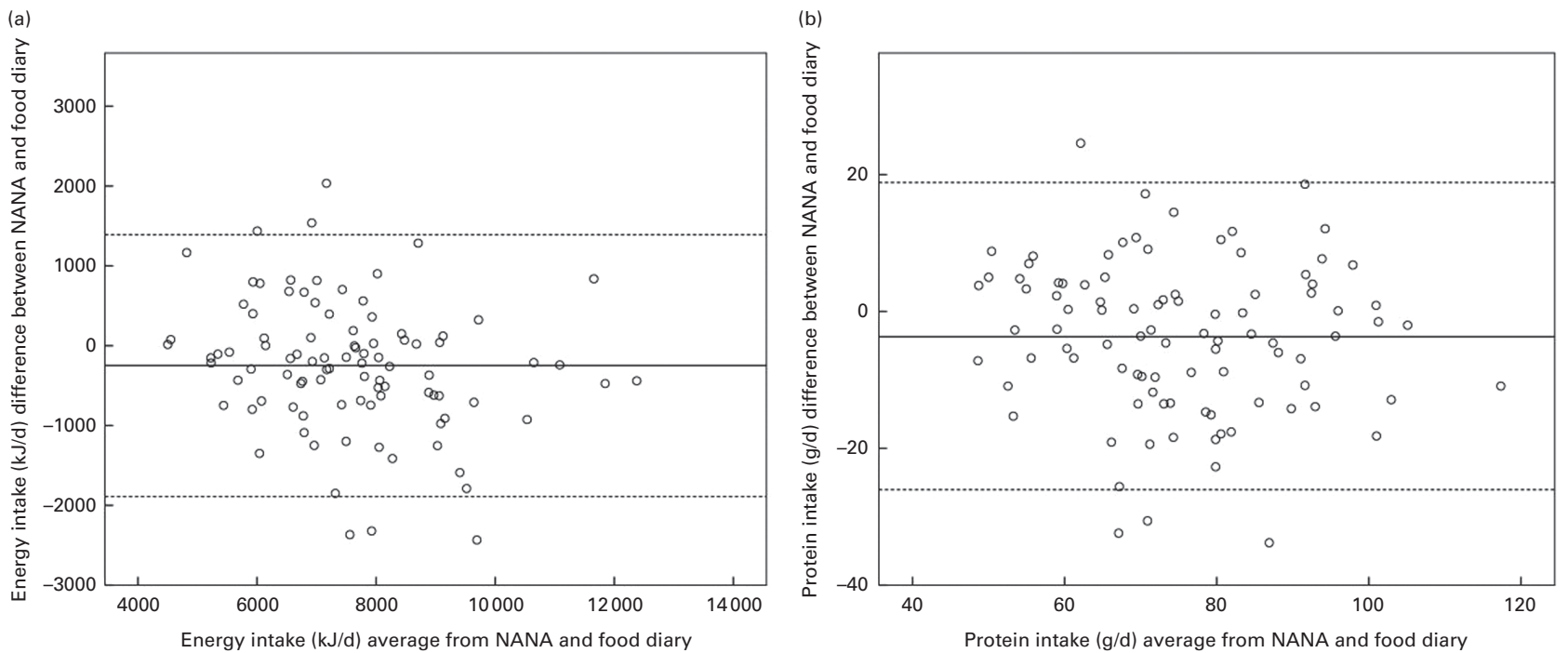

(c)

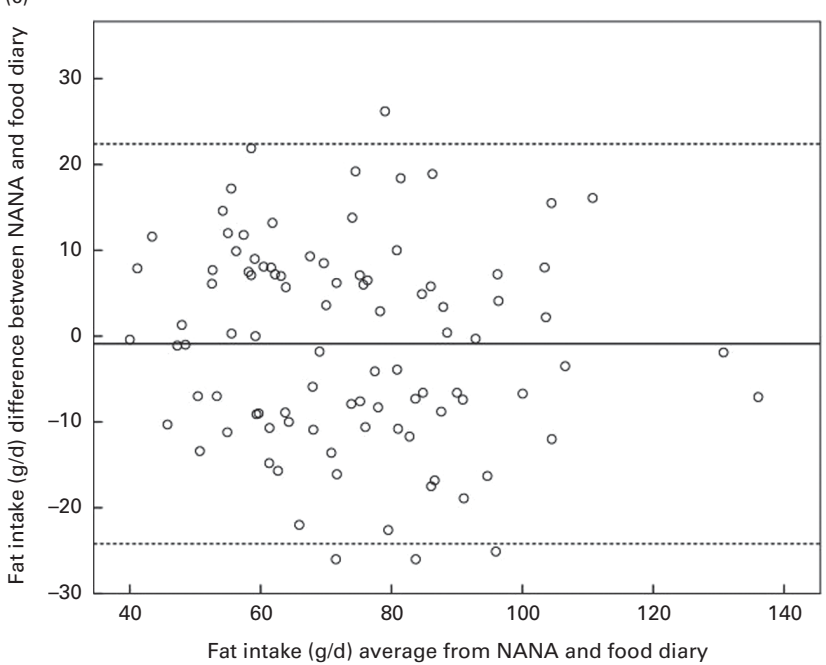

(d)

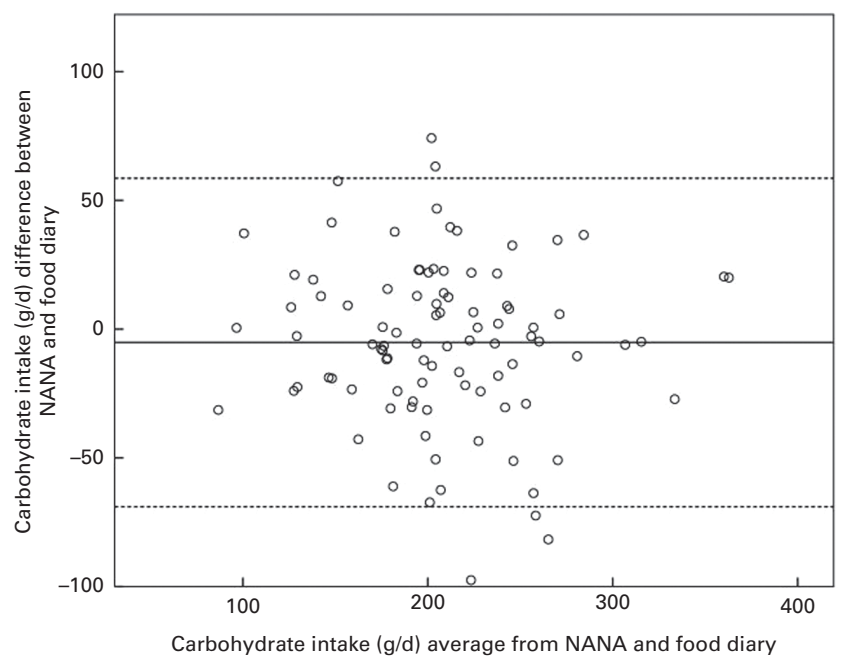

Fig. 3. Bland-Altman plots of the difference between intakes recorded by the Novel Assessment of Nutrition and Ageing (NANA) method and that recorded by the $4 \mathrm{~d}$ estimated food diary against the mean intakes for the two reporting methods for energy (a), protein (b), fat (c) and carbohydrate (d). Mean difference ( - ) and limits of agreement $(2 \mathrm{sD})(---)$ for nutrient intakes were, respectively, as follows: energy $-249 \mathrm{~kJ} / \mathrm{d},-1887 / 1389 \mathrm{~kJ} / \mathrm{d}$; protein $-3.68 \mathrm{~g} / \mathrm{d}$, $-26.04 / 18.68 \mathrm{~g} / \mathrm{d}$; fat $-0.882 \mathrm{~g} / \mathrm{d},-24.2 / 22.4 \mathrm{~g} / \mathrm{d}$; carbohydrate $-5.18 \mathrm{~g} / \mathrm{d},-68.98 / 58.62 \mathrm{~g} / \mathrm{d}$.

Independent biomarkers of dietary intake are currently limited in their scope, but those that are available have provided an unbiased and objective method to validate dietary intake $^{(35)}$. Here, we used urinary urea excretion and plasma ascorbic acid as the independent biomarkers of protein and vitamin $\mathrm{C}$ intakes, respectively. The significant correlations between dietary protein intake and the independent biomarker of protein intake for both the food diary and the NANA method suggest that both reporting methods are valid and comparable in their assessment of dietary protein intake. The strength of the correlation was comparable with that reported in the literature for a single observation ${ }^{(36)}$. A significant correlation was also observed between plasma ascorbic acid and dietary vitamin $\mathrm{C}$ intake as assessed using the NANA method, but not with the food diary. This observation provides further confidence that NANA is an accurate method of dietary assessment. The different timings of the dietary recording methods in relation to the blood sample collection may account for the lack of the association between plasma ascorbic acid and dietary vitamin $\mathrm{C}$ intake as assessed by the food diary method. The blood sample for ascorbic acid analysis was taken within a week of the NANA data recording period, but more than a month before the food diary was recorded.

The vast majority of the participants complied well with all the aspects of the studies; however, a small minority $(6.8 \%)$ of the older adults recruited to the studies failed to use the NANA system as instructed and were excluded from the analysis. In all instances, this was reflected in a lack of recordings on the NANA system. This small failure rate is likely to be due to a combination of factors, including study fatigue associated with the long recording period (3 weeks) in study 1 , failure to adequately record foods consumed outside the home, and for one individual an apparent failure to 

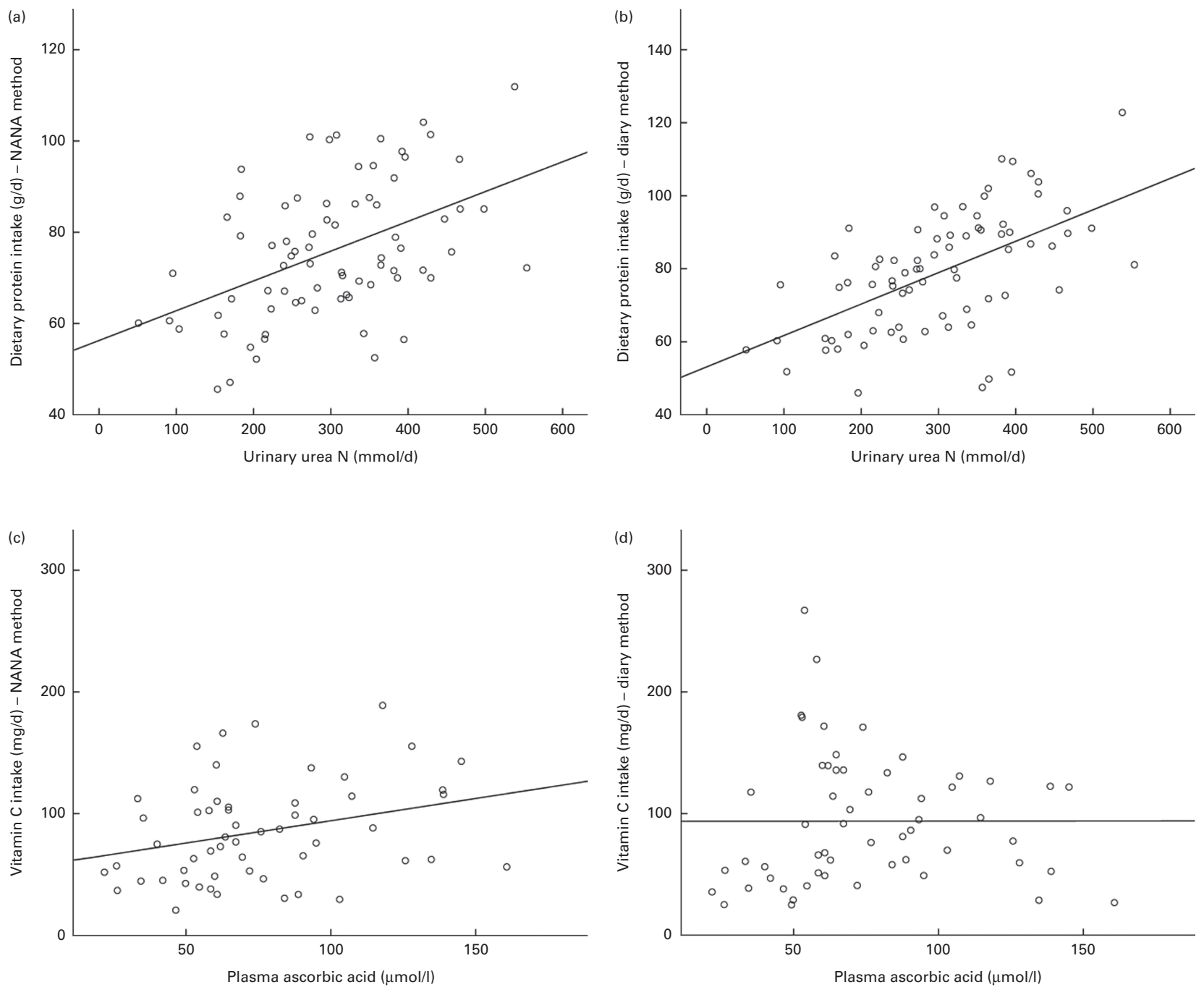

Fig. 4. Scatter plots of the relationship between urinary urea excretion and dietary protein intake recorded by the Novel Assessment of Nutrition and Ageing (NANA) method (a) and that recorded by the $4 \mathrm{~d}$ estimated food diary (b), and between plasma ascorbic acid and dietary vitamin $\mathrm{C}$ intake recorded by the NANA method (c) and the $4 \mathrm{~d}$ estimated food diary (d).

understand the instructions given. This proportion of withdrawals and exclusions is comparable with that reported in other studies ${ }^{(23,37)}$ and does not detract from the evidence that the NANA method was acceptable to the majority of the participants.

The present study demonstrates the feasibility of using a self-administered computer-based dietary assessment method with older adults. It is important to acknowledge that the majority of the participants recruited to the trial appeared to be comfortable with a variety of technologies, including a higher than anticipated use of the Internet. However, computer and Internet usage is known to be increasing in the older adult population ${ }^{(38)}$ and computer-based dietary assessment is likely to become an increasingly viable option for this age group as the computer-literate population continues to age. It is important to note that the small proportion of the population used in the present study, who had never previously used a computer, was able to use the touchscreen computer. This may be a reflection of the thorough one-to-one training given to each participant at the installation of the NANA system.

A particular strength of the present study is that the NANA method was validated in the real world rather than in an artificial laboratory environment. The present study demonstrates that older adult participants were willing to use the NANA method for periods ranging from 1 to 3 weeks, suggesting that the method is amenable to repeated measures, and for longitudinal assessment. Anecdotally, many participants reported the ease at which they were able to incorporate the use of the NANA method into their everyday life. We believe that the success of the NANA adoption by the participants was in a large part due to the initial user-centred development of NANA and the iterative input of older adults into the design of the software. We acknowledge that, as with most research studies, the participants in the study were generally interested in research and were well motivated, and may not be representative of the more general older adult population. 
The main advantage of the NANA method over most traditional methods of dietary assessment is that capture of dietary intake information is, in the main, done when food and drink is about to be consumed and is coupled with a photographic record of the food. Portion size assessment, thus, moves from the participant to the researcher, reducing the need for lengthy and burdensome participant interviews and potentially improving the accuracy of portion size assessment. This approach is particularly advantageous for older adults who encounter difficulties with retrospective methods of dietary assessment ${ }^{(3)}$. A further advantage of the NANA method is that data collection is self-administered and is independent of an interviewer, thus reducing the possible impact of interviewer bias.

Older adults represent a hugely heterogeneous population with an age range that spans approximately 40 years and with a range of functional abilities. A limitation of the study is that the average age of the participants was 73 years. Only six of the participants were over the age of 80 years, with only two over the age of 85 years. From such a small sample, it is not possible to conclude that NANA would be a suitable method for dietary assessment in the oldest age category of individuals who are most likely to encounter physical and cognitive frailty, although the results are promising.

There are several developments that could be made to increase the potential application and ease of NANA use. First, in its current format, the NANA software provides a description and a photograph of the food consumed, and the nutritional analysis of this information remains the responsibility of the researcher. A future development of this method would be automated dietary assessment in which nutrient composition data would be automatically assigned to foods upon selection, thereby circumventing the need for independent nutrient analysis software. Such an approach would significantly streamline the data collection and analysis process; however, as with most automated dietary assessment methods, the principal challenge would be the accurate assessment of food portion size. Automated estimation of food portion size from digital images is currently being developed and may provide a viable option in the future ${ }^{(11)}$. Second, the NANA method was developed and validated with and for the community-living UK Caucasian older adult population. The range of food items available for the user to select reflects the range of foods commonly consumed by this population. It is anticipated that the hierarchical food tree would require expansion to allow the capture of dietary information from ethnic minorities or from populations living outside of the UK. Similarly, the food tree would need to be adapted if the NANA method of dietary assessment were to be applied to younger age groups or to older adults living in institutions. Finally, a current limitation of the NANA method is the necessity to provide the touch-screen computer hardware to the users, which could have significant cost and logistical implications for larger studies. Tablet computers offer a viable alternative, and we are currently exploring the feasibility of using them for dietary assessment in older adults. Since the cost of tablet computers is dropping rapidly, this approach may offer a cost-effective alternative to existing methods of dietary assessment in the near future, and as the adoption of touch-screen tablet computers by the general population increases, the need for researchers to supply the hardware is likely to decrease. The application of the NANA method to a lightweight, portable tablet computer also has an additional advantage in that it could enable the direct recording of food eaten outside of the home, which could further improve the accuracy of the NANA method.

\section{Conclusion}

In the present study, we have assessed the relative validity of the NANA method for the assessment of dietary intake in older adults. The NANA method compares well with the $4 \mathrm{~d}$ estimated food diary and offers a potential alternative to the food diary for the estimation of dietary intake in communityliving older adults. There is scope for further development and improvement of the method including automated dietary assessment and the transfer of the software to tablet computers that would facilitate larger-scale population-based studies. We are also currently exploring the feasibility of using the NANA method to assess the dietary intake of nursing home residents where carers will be asked to interact with the software on behalf of the residents. Most importantly, we have demonstrated the feasibility of the self-administered computer-based dietary assessment method in older adults, and hope that this will encourage other researchers to adopt similar approaches in the future.

\section{Acknowledgements}

The authors thank the participants for their involvement in the study. The authors also gratefully acknowledge Laura Brown, Hassan Khandra, Alan Godfrey and Rebecca Rowland-Jones for their contribution to the developmental aspects of the NANA system, Marilyn Hill for assistance with the biochemical analysis and Margo Barker for useful advice throughout the study.

The present study was funded by the Economic and Social Research Council (ESRC), New Dynamics of Ageing Programme (RES-354-25-0003). The ESRC had no role in the design and analysis of the study or in the writing of this article.

The authors declare that there are no conflicts of interest.

The authors' contributions are as follows: A. J. A., F. H., T. D. A. and E. A. W. conceived the project; C. M. T., S. E. F., A. J. A., F. H., T. D. A. and E. A. W. contributed to the study design; T. S. and F. H. developed the software; C. M. T., L. M., S. E. F., D. S. and E. A. W. collected the data; C. M. T. and E. A. W. performed the data analysis; E. A. W. drafted the manuscript. All authors approved the final content of the manuscript.

\section{References}

1. Roberts CK \& Barnard RJ (2005) Effects of exercise and diet on chronic disease. J Appl Physiol 98, 3-30. 
2. Dwyer J (2006) Starting down the right path: nutrition connections with chronic diseases of later life. Am J Clin Nutr 83, 415S-420S.

3. van Staveren WA, de Groot LC, Blauw YH, et al. (1994) Assessing diets of elderly people: problems and approaches. Am J Clin Nutr 59, 221S-223S.

4. McNeill G, Winter J \& Jia X (2009) Diet and cognitive function in later life: a challenge for nutrition epidemiology. Eur J Clin Nutr 63, S33-S37.

5. de Vries JHM, de Groot LCPGM \& van Staveren WA (2009) Dietary assessment in elderly people: experiences gained from studies in the Netherlands. Eur J Clin Nutr 63, S69-S74.

6. Stratton RJ, King CL, Stroud MA, et al. (2006) 'Malnutrition Universal Screening Tool' predicts mortality and length of hospital stay in acutely ill elderly. Br J Nutr 95, 325-330.

7. Vellas B, Guigoz Y, Baumgartner M, et al. (2000) Relationships between nutritional markers and the mini-nutritional assessment in 155 older persons. J Am Geriatr Soc 48, $1300-1309$.

8. Ortiz-Andrellucchi A, Sánchez-Villegas A, Doreste-Alonso J, et al. (2009) Dietary assessment methods for micronutrient intake in elderly people: a systematic review. $\mathrm{Br} J \mathrm{Nutr}$ 102, S118-S149.

9. Ngo J, Engelen A, Molag M, et al. (2009) A review of the use of information and communication technologies for dietary assessment. Br J Nutr 101, S102-S112.

10. Illner A-K, Freisling H, Boeing H, et al. (2012) Review and evaluation of innovative technologies for measuring diet in nutritional epidemiology. Int J Epidemiol 41, 1187-1203.

11. Stumbo PJ (2013) New technology in dietary assessment: a review of digital methods in improving food record accuracy. Proc Nutr Soc 72, 70-76.

12. Slimani N, Deharveng G, Charrondière RU, et al. (1999) Structure of the standardized computerized 24-h diet recall interview used as reference method in the 22 centers participating in the EPIC project. Comput Methods Programs Biomed 58, 251-266.

13. Subar A, Kirkpatrick S, Mittl B, et al. (2012) The Automated Self-Administered 24-Hour Dietary Recall (ASA24): a resource for researchers, clinicians, and educators from the National Cancer Institute. J Acad Nutr Diet 112, 1134-1137.

14. Arab L, Tseng C, Ang A, et al. (2011) Validity of a multipass, web-based, 24-hour self-administered recall for assessment of total energy intake in blacks and whites. Am J Epidemiol 174, 1256-1265.

15. Foster E, Hawkins A, Delve J, et al. (2013) Reducing the cost of dietary assessment: Self-Completed Recall and Analysis of Nutrition for use with children (SCRAN24). J Hum Nutr Diet 27, Suppl. 1, 26-35.

16. Apovian CM, Murphy MC, Cullum-Dugan D, et al. (2010) Validation of a web-based dietary questionnaire designed for the DASH (Dietary Approaches to Stop Hypertension) diet: the DASH Online Questionnaire. Public Health Nutr 13, 615-622.

17. Slattery ML, Murtaugh MA, Schumacher MC, et al. (2008) Development, implementation, and evaluation of a computerized self-administered diet history questionnaire for use in studies of American Indian and Alaskan native people. J Am Diet Assoc 108, 101-109.

18. Beasley JM, Davis A \& Riley WT (2009) Evaluation of a webbased, pictorial diet history questionnaire. Public Health Nutr 12, 651-659.

19. Edwards SL, Slattery ML, Murtaugh MA, et al. (2007) Development and use of touch-screen audio computer-assisted self-interviewing in a study of American Indians. $A m J$ Epidemiol 165, 1336-1342.

20. Matthys C, Pynaert I, De Keyzer W, et al. (2007) Validity and reproducibility of an adolescent web-based food frequency questionnaire. J Am Diet Assoc 107, 605-610.

21. Wong SS, Boushey CJ, Novotny R, et al. (2008) Evaluation of a computerized food frequency questionnaire to estimate calcium intake of Asian, Hispanic, and non-Hispanic white youth. J Am Diet Assoc 108, 539-543.

22. Vereecken CA, De Bourdeaudhuij I \& Maes L (2010) The HELENA online food frequency questionnaire: reproducibility and comparison with four 24-h recalls in Belgian-Flemish adolescents. Eur J Clin Nutr 64, 541-548.

23. Labonte ME, Cyr A, Baril-Gravel L, et al. (2012) Validity and reproducibility of a web-based, self-administered food frequency questionnaire. Eur J Clin Nutr 66, 166-173.

24. McCabe-Sellers B (2010) Advancing the art and science of dietary assessment through technology. J Am Diet Assoc 110, $52-54$.

25. Vereecken CA, Dohogne S, Covents M, et al. (2010) How accurate are adolescents in portion-size estimation using the computer tool Young Adolescents' Nutrition Assessment on Computer (YANA-C). Br J Nutr 103, 1844-1850.

26. Touvier M, Kesse-Guyot E, Méjean C, et al. (2011) Comparison between an interactive web-based self-administered $24 \mathrm{~h}$ dietary record and an interview by a dietitian for large-scale epidemiological studies. Br J Nutr 105, 1055-1064.

27. Adamson AJ, Collerton J, Davies K, et al. (2009) Nutrition in advanced age: dietary assessment in the Newcastle $85+$ study. Eur J Clin Nutr 63, S6-S18.

28. Nelson M, Atkinson M \& Meyer J (1997) A Photographic Atlas of Food Portion Sizes. London: MAFF Publications.

29. Vuilleumier J \& Keck E (1989) Fluorometric assay of vitamin $C$ in biological materials using a centrifugal analyser with fluorescence attachment. J Micronutr Anal 5, 25-34.

30. Bingham S, Williams R, Cole T, et al. (1988) Reference values for analytes of 24-h urine collections known to be complete. Ann Clin Biochem 25, 610-619.

31. Bland J \& Altman D (1986) Statistical methods for assessing agreement between two methods of clinical measurement. Lancet $\mathbf{i}, 307-310$.

32. Kikunaga S, Tin T, Ishibashi G, et al. (2007) The application of a handheld personal digital assistant with camera and mobile phone card (Wellnavi) to the general population in a dietary survey. J Nutr Sci Vitaminol (Tokyo) 53, 109-116.

33. Margetts B \& Nelson M (1997) Design Concepts in Nutritional Epidemiology. New York: Oxford University Press.

34. Poslusna K, Ruprich J, de Vries JHM, et al. (2009) Misreporting of energy and micronutrient intake estimated by food records and 24 hour recalls, control and adjustment methods in practice. Br J Nutr 101, S73-S85.

35. Hedrick V, Dietrich A, Estabrooks P, et al. (2012) Dietary biomarkers: advances, limitations and future directions. Nutr J 11, 109.

36. Bingham SA (2003) Urine nitrogen as a biomarker for the validation of dietary protein intake. J Nutr 133, 921S-924S.

37. Probst YC, Faraji S, Batterham M, et al. (2008) Computerized dietary assessments compare well with interviewer administered diet histories for patients with type 2 diabetes mellitus in the primary healthcare setting. Patient Educ Couns $\mathbf{7 2}$, 49-55.

38. Ofcom (2013) Adults' media use and attitudes report 2013. http://stakeholders.ofcom.org.uk/market-data-research/ media-literacy/media-lit-research/adults-2013/ 